\title{
Otimalidade Inversa em Controle Extremal com Atrasos
}

\author{
Denis César Ferreira* Tiago Roux Oliveira* \\ * Departamento de Engenharia Eletrônica e Telecomunicações \\ Universidade do Estado do Rio de Janeiro (UERJ) \\ Rio de Janeiro, RJ 20550-900,Brasil.(e-mail: denistcf@yahoo.com.br, \\ tiagoroux@uerj.br)
}

\begin{abstract}
We present a Gradient-based extremum seeking algorithm for maximizing unknown maps in the presence of constant delays. It is corporated a filtered predictor feedback with a perturbation-based estimate for the Hessian of locally quadratic maps. Exponential stability and convergence to a small neighborhood of the unknown extremum point are achieved by using backstepping transformation and averaging theory in infinite dimensions. The low-pass filter (with a high enough pole) in the predictor feedback allows the technical application of the Hale and Lunel's averaging theorem for functional differential equations and also establishes an inverse optimal result for the closed-loop system. This inverse optimality property is for the first time demonstrated in extremum seeking designs and justifies the heuristic use of a low-pass filter between the demodulation and the integrator, which has historically been a part of the extremum seeking implementations free of delays.

Resumo: Apresenta-se um algoritmo de busca extremal baseado no Gradiente para maximizar mapas desconhecidos na presença de atrasos. Introduz-se uma versão filtrada do preditor baseado em estimativa local da Hessiana para mapas quadráticos. A estabilidade exponencial e a convergência para uma pequena vizinhança do ponto extremo desconhecido são alcançadas utilizando a transformação backstepping e a teoria da média em dimensões infinitas. O filtro passa-baixa (com um polo suficientemente alto) na realimentação do preditor permite aplicação técnica do teorema da média de Hale e Lunel para equações diferenciais funcionais e também estabelece o resultado da otimalidade inversa para o sistema de malha fechada. Esta propriedade de otimalidade inversa é pela primeira vez demonstrada em projetos de busca extremal e justifica o uso heurístico de um filtro passa-baixa entre o sinal de demodulação e o integrador, que tem sido historicamente utilizado em implementações de busca extremal sem atrasos.
\end{abstract}

Keywords: inverse optimality, time delay, adaptive control, extremum seeking, predictors, backstepping transformation, averaging in infinite dimensions.

Palavras-chaves: otimalidade inversa, atrasos, controle adaptativo, busca extremal, preditores, transformação backstepping, teoria da média em dimensões infinitas.

\section{INTRODUÇÃO}

O Controle por Busca Extremal (Extremum Seeking Control - ESC) é um método robusto de otimização que tem a capacidade de determinar o extremo de um mapa nãolinear de desempenho de uma planta (Krstić and Wang, 2000). O ESC não possui a obrigatoriedade do conhecimento explícito da planta nem da função que se pretende otimizar, desde que se tenha o conhecimento de que possua um extremo (Krstić, 2009). O que irá definir se o mapa estático se trata de uma função de máximo ou de mínimo é o sinal da Hessiana.

No controle por busca extremal existem vários trabalhos que aplicam filtros passa-alta e passa-baixa para melhorar o ajuste/sintonia dos parâmetros do controlador e o desempenho do sistema em malha fechada. Entretanto, os mesmos não apresentam nenhuma sustentação teórica que justifique a inclusão dos filtros. (Krstić, 2014; Adetola and Guay, 2007; Tan et al., 2009; Nesić et al., 2010; Ghaffari et al., 2012; Liu and Krstić, 2012; Oliveira et al., 2011, 2012), pelo contrário, apenas argumentações heurísticas são levantadas.

Neste trabalho será discutida pela primeira vez na literatura a prova da otimalidade inversa e sua influência no controle por busca extremal na presença de atrasos (embora os resultados também sejam válidos no caso livre de atrasos). A otimalidade inversa foi definida em (Kalman, 1964) da seguinte forma: "Dado um sistema dinâmico e uma lei de controle conhecida, encontre critérios de desempenho (se houver) para os quais essa lei de controle é ótima". A otimalidade inversa é garantida quando um controlador estabilizante é ótimo e, para uma dada função de Lyapunov, é possível construir uma realimentação que é ótima com respeito a alguma função custo. Em geral, esse funcional inclui uma penalidade no esforço de controle (ou sua derivada) e tem margem de ganho infinita. Resultados e simulação ilustram numericamente as vantagens de se satisfazer a otimalidade inversa. O artigo está organizado 
da seguinte forma. Na Seção 2 o controle por busca extremal com atraso é apresentado. Na Seção 3 a prova da otimalidade é desenvolvida juntamente com a análise de estabilidade, enquanto que na Seção 4 apresenta-se as simulações das variáveis do ESC verificando-se a otimalidade inversa no sistema discutido. Por fim a Seção 5 conclui o artigo indicando trabalhos futuros.

Notações e Normas: A norma-2 de um vetor de estado $X(t)$ de dimensão finita de uma equação diferencial ordinária (EDO) é denotada por barras simples, $|X(t)|$. Em contraste, as normas funções (de $x$ ) são denotadas por barras duplas. Por padrão, $\|\cdot\|$ denota a norma espacial $L_{2}[0, D]$, i.e., $\|\cdot\|=\|\cdot\|_{L_{2}[0, D]}$. Como a variável de estado $u(x, t)$ da equação diferencial parcial (EDP) é uma função de dois argumentos, deve-se dar enfâse que levando em conta a norma de uma das variáveis faz-se à norma uma função da outra variável, assim como adotado em (Krstić, 2009). Por exemplo, a norma $L_{2}[0, D]$ de $u(x, t)$ em $x \in[0, D]$ é $\|u(t)\|=\left(\int_{0}^{D} u^{2}(x, t) d x\right)^{1 / 2}$.

\section{CONTROLE POR BUSCA EXTREMAL COM ATRASOS}

O controle por busca extremal escalar considera aplicações em que o objetivo é maximizar (ou minimizar) a saída $y \in R$ de um mapeamento não-linear estático variando-se a entrada $\theta \in R$. Neste exemplo assume-se adicionalmente que existe um atraso constante $e$ desconhecido $D \geq 0$ no caminho de atuação ou no sitema de medição tal que a saída mensurada é dada por

$$
y(t)=Q(\theta(t-D)) .
$$

A fim de facilitar a notação, assume-se ao longo do artigo que o sistema tem sua saída atrasada de acordo com o diagrama de blocos na Figura 1. Entretanto, os resultados neste artigo podem ser diretamente estendidos para o caso da entrada atrasada, visto que qualquer atraso na entrada pode ser movido para a saída do mapa estático. O caso onde atrasos de entrada $D_{\text {in }}$ e de saída $D_{\text {out }}$ ocorrem simultamente pode também ser tratado assumindo que o atraso total a ser neutralizado é $D=D_{\text {in }}+D_{\text {out }}$, com $D_{\text {in }}, D_{\text {out }} \geq 0$. Sem perda de generalidade, considera-se o problema de busca pelo máximo, tal que o valor de $\theta$ que maximiza $y$ é denotado por $\theta^{*}$. Por simplicidade, assumese que o mapa não-linear é quadrático:

$$
Q(\theta)=y^{*}+\frac{H}{2}\left(\theta-\theta^{*}\right)^{2}
$$

onde além das constantes $\theta^{*} \in R$ e $y^{*} \in R$ serem desconhecidas, o escalar $H<0$ é a Hessiana desconhecida do mapa estático. Substituindo-se (2) em (1), obtém-se o mapa quadrático e estático com atraso de interesse:

$$
y(t)=y^{*}+\frac{H}{2}\left(\theta(t-D)-\theta^{*}\right)^{2} .
$$

\subsection{Sinais do Sistema}

Seja $\hat{\theta}$ a estimativa de $\theta^{*}$ e

$$
\tilde{\theta}(t)=\hat{\theta}(t)-\theta^{*}
$$

o erro de estimativa. Da Figura 1, a dinâmica do erro pode ser escrita como

$$
\dot{\tilde{\theta}}(t-D)=U(t-D)
$$

Além disso, tem-se que

$$
G(t)=M(t) y(t), \quad \theta(t)=\hat{\theta}(t)+S(t),
$$

onde os sinais de perturbações são redefinidos por

$$
S(t)=a \sin (\omega(t+D)), \quad M(t)=\frac{2}{a} \sin (\omega t),
$$

como a amplitude $a>0$ e a frequência $\omega>0$. O sinal

$$
\hat{H}(t)=N(t) y(t)
$$

é aplicado para se obter uma estimativa da Hessiana $H$ desconhecida, onde o sinal de demodulação $N(t)$ é dado por

$$
N(t)=-\frac{8}{a^{2}} \cos (2 \omega t) .
$$

Em (Ghaffari et al., 2012), foi provado que

$$
\frac{1}{\Pi} \int_{0}^{\Pi} N(\sigma) y d \sigma=H, \quad \Pi=2 \pi / \omega,
$$

se um mapa quadrático como em (2) é considerado. Em outras palavras, a versão média de $\hat{H}(t)$ é dada por $\hat{H}_{\mathrm{av}}=$ $(N y)_{\mathrm{av}}=H$.

\subsection{Realimentação por Preditor com Estimativa da Hessiana}

Utilizando-se a análise média (averaging analysis) pode-se verificar que a versão média do sinal $G(t)$ em (6) é dada por

$$
G_{\mathrm{av}}(t)=H \tilde{\theta}_{\mathrm{av}}(t-D) .
$$

De (5), os seguintes modelos médios podem ser obtidos

$$
\begin{gathered}
\dot{\tilde{\theta}}_{\mathrm{av}}(t-D)=U_{\mathrm{av}}(t-D), \\
\dot{G}_{\mathrm{av}}(t)=H U_{\mathrm{av}}(t-D),
\end{gathered}
$$

onde $U_{\text {av }} \in R$ é o controle médio resultante para $U \in R$.

No sentido de motivar o projeto de controle por preditor, a ideia aqui é compensar o atraso pela realimentação do estado futuro $G(t+D)$, ou $G_{\mathrm{av}}(t+D)$ no sistema médio equivalente. Dado qualquer ganho de estabilização $k>0$ para o sistema não atrasado, deseja-se ter uma lei de controle tal que

$$
U_{\mathrm{av}}(t)=k G_{\mathrm{av}}(t+D), \quad \forall t \geq 0,
$$

o que parece ser não implementável já que ela requer valores futuros do estado. No entanto, aplicando a fórmula da variação das contantes para (13) pode-se expressar o estado futuro como

$$
G_{\mathrm{av}}(t+D)=G_{\mathrm{av}}(t)+H \int_{t-D}^{t} U_{\mathrm{av}}(\sigma) d \sigma,
$$

que fornece o estado futuro $G_{\mathrm{av}}(t+D)$ em termos do sinal de controle médio $U_{\text {av }}(\sigma)$ na janela causal de tempo $[t-D, t]$. Isto produz a lei de realimentação:

$$
U_{\mathrm{av}}(t)=k\left[G_{\mathrm{av}}(t)+H \int_{t-D}^{t} U_{\mathrm{av}}(\sigma) d \sigma\right] .
$$

Portanto, de (15) e (16), a lei de realimentação média (14) pode ser obtida de fato como desejada. Consequentemente,

$$
\dot{\tilde{\theta}}_{\mathrm{av}}(t)=k G_{\mathrm{av}}(t+D), \quad \forall t \geq 0 .
$$




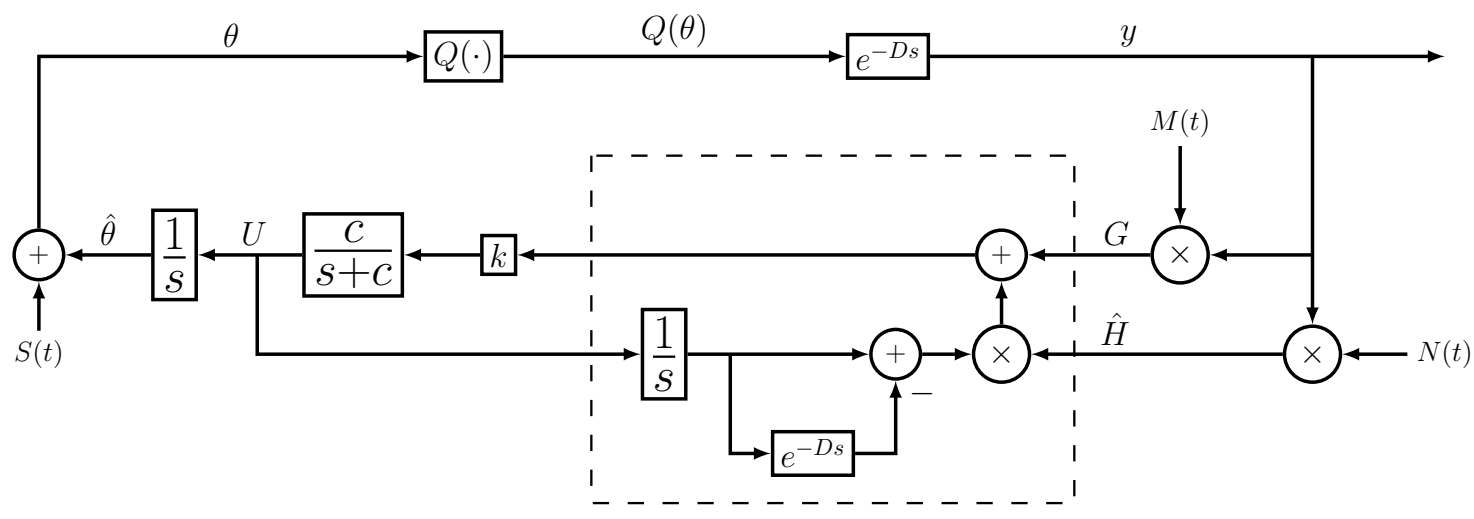

Figura 1. Diagrama de blocos do esquema de predição básico para compensação do atraso no ESC gradiente. A realimentação por preditor com a Hessiana baseada em perturbação e averaging obedece a equação(19). Os sinais de perturbação senoidal são dados por $S(t)=a \sin (\omega(t+D))$ e $M(t)=\frac{2}{a} \sin (\omega t)$ e o sinal de demodulação é $N(t)=-\frac{8}{a^{2}} \cos (2 \omega t)$.

Dessa forma, a partir de (11), tem-se

$$
\frac{d \tilde{\theta}_{\mathrm{av}}(t)}{d t}=k H \tilde{\theta}_{\mathrm{av}}(t), \quad \forall t \geq D
$$

com um equilíbrio $\tilde{\theta}_{\mathrm{av}}^{e}=0$ exponencialmente atrativo, uma vez que $k>0$ no projeto do controlador e $H<0$ por hipótese.

O desempenho do controle pode ser melhorado aplicandose o conceito de otimalidade inversa, onde um filtro passabaixas é inserido no controlador baseado em preditor. Neste caso, propõe-se a seguinte versão filtrada do preditor de dimensão infinita para a compensação do atraso (Krstić, 2008).

$$
U(t)=\frac{c}{s+c}\left\{k\left[G(t)+\hat{H}(t) \int_{t-D}^{t} U(\tau) d \tau\right]\right\},
$$

onde $c>0$ é suficientemente grande. A versão média do sinal (19) é um sinal filtrado de (16). Esta filtragem passa-baixa é particularmente necessária na análise de estabilidade quando o teorema da média em dimensões infinitas (Hale and Lunel, 1990; Lehman, 2002) é invocado.

\section{ANÁLISE DE ESTABILIDADE E OTIMALIDADE INVERSA}

Nesta seção, a análise da estabilidade é realizada e a prova da otimalidade inversa é apresentada.

Teorema 1 Existe um $c^{*}$ tal que o sistema médio realimentado (13) e (19) é exponencialmente estável no sentido da norma

$$
\Psi(t)=\left(\left|\tilde{\theta}_{\mathrm{av}}(t-D)\right|^{2}+\int_{t-D}^{t} U_{\mathrm{av}}(\tau)^{2} d \tau+U_{\mathrm{av}}(t)^{2}\right)^{1 / 2}
$$

para todo $c>c^{*}$. Além disso, existe $c^{* *}>c^{*}$ tal que, para qualquer $c \geq c^{* *}$, a lei de controle (19) minimiza a função custo

$$
\left.J=\int_{0}^{\infty}(\mathcal{L}(t))+\dot{U}_{\mathrm{av}}(t)^{2}\right) d t
$$

onde $\mathcal{L}(t)$ é um funcional de $\left(\tilde{\theta}_{\mathrm{av}}(t-D), U(\tau)\right), \tau \in$ $[t-D, t]$ tal que

$$
\mathcal{L}(t) \geq \mu \Psi(t)^{2}
$$

para algum $\mu(c)>0$ com a propriedade que $\mu(c) \rightarrow \infty \grave{a}$ medida que $c \rightarrow \infty$.

Prova: A demonstração segue os passos de 1 a 7 abaixo.

Passo 1: EDP para Representação do Atraso

De acordo com (Krstić, 2009), o atraso em (5) pode ser representado usando uma EDP de transporte como

$$
\begin{aligned}
\dot{\tilde{\theta}}(t-D) & =u(0, t), \\
u_{t}(x, t) & =u_{x}(x, t), \quad x \in[0, D], \\
u(D, t) & =U(t),
\end{aligned}
$$

onde a solução de $(24)-(25)$ é

$$
u(x, t)=U(t+x-D) .
$$

\section{Passo 2: Equações em Malha Fechada}

Primeiramente, substituindo-se $S(t)$ dado em (7) em $\theta(t)$ na equação (6), obtém-se

$$
\theta(t)=\hat{\theta}(t)+a \sin (\omega(t+D)) .
$$

Agora, colocando-se (4) e (27) em (3) tal que a saída seja dada em termos de $\tilde{\theta}$ :

$$
y(t)=y^{*}+\frac{H}{2}(\tilde{\theta}(t-D)+a \sin (\omega t))^{2} .
$$

Substituindo-se $M(t)$ definido por $(7)$ em $G(t)$ dado por (6), além disso (9) em (8) e representando o integrando em (19) usando o estado da EDP de transporte, obtém-se

$$
\begin{gathered}
U(t)=\frac{c}{s+c}\left\{k\left[G(t)+\hat{H}(t) \int_{0}^{D} u(\sigma, t) d \sigma\right]\right\}, \\
G(t)=\frac{2}{a} \sin (\omega t) y(t), \\
\hat{H}(t)=-\frac{8}{a^{2}} \cos (2 \omega t) y(t) .
\end{gathered}
$$

Posteriormente, substituindo-se (28) em (30) e (31), e então depois o resultado de (30) e (31) em (29), assim 
como extraindo o fator comum $y$ na versão resultante de (29), tem-se

$$
\begin{aligned}
U(t) & =\frac{c}{s+c}\left\{k\left[y^{*}+\frac{H}{2}(\tilde{\theta}(t-D)+a \sin (\omega t))^{2}\right]\right. \\
& \left.\times\left[\frac{2}{a} \sin (\omega t)-\frac{8}{a^{2}} \cos (2 \omega t) \int_{0}^{D} u(\sigma, t) d \sigma\right]\right\} .
\end{aligned}
$$

Expandindo-se o binômio em (32), obtém-se

$$
\begin{aligned}
& U(t)=\frac{c}{s+c}\left\{k \left[y^{*}+\frac{H}{2} \tilde{\theta}^{2}(t-D)\right.\right. \\
& \left.+H a \sin (\omega t) \tilde{\theta}(t-D)+\frac{a^{2} H}{2} \sin ^{2}(\omega t)\right] \\
& \left.\times\left[\frac{2}{a} \sin (\omega t)-\frac{8}{a^{2}} \cos (2 \omega t) \int_{0}^{D} u(\sigma, t) d \sigma\right]\right\} .
\end{aligned}
$$

Finalmente, substituindo (33) em (25), pode-se reescrever (23)-(25) como

$$
\begin{aligned}
& \dot{\tilde{\theta}}(t-D)=u(0, t), \\
& \partial_{t} u(x, t)=\partial_{x} u(x, t), \quad x \in[0, D], \\
& u(D, t)=\frac{c}{s+c}\left\{k \left[y^{*}+\frac{H}{2} \tilde{\theta}^{2}(t-D)+\right.\right. \\
& \left.H a \sin (\omega t) \tilde{\theta}(t-D)+\frac{a^{2} H}{2} \sin ^{2}(\omega t)\right] \\
& \left.\times\left[\frac{2}{a} \sin (\omega t)-\frac{8}{a^{2}} \cos (2 \omega t) \int_{0}^{D} u(\sigma, t) d \sigma\right]\right\}_{0}^{(35)} \\
& =\frac{c}{s+c}\left\{k \left[y^{*} \frac{2}{a} \sin (\omega t)-y^{*} \frac{8}{a^{2}} \cos (2 \omega t) \int_{0}^{D} u(\sigma, t) d \sigma\right.\right. \\
& +\frac{H}{a} \tilde{\theta}^{2}(t-D) \sin (\omega t) \\
& -\frac{4 H}{a^{2}} \tilde{\theta}^{2}(t-D) \cos (2 \omega t) \int_{0}^{D} u(\sigma, t) d \sigma \\
& +2 H \sin ^{2}(\omega t) \tilde{\theta}(t-D) \\
& +[H+H \cos (4 \omega t)] \int_{0}^{D} \sin ^{D}(\omega t) \tilde{\theta}(t-D) \cos (2 \omega t) \int_{0}^{D} u(\sigma, t) d \sigma \\
& +\frac{3 a H}{a} \sin (\omega t)-\frac{a H}{4} \sin (3 \omega t)-2 H \cos (2 \omega t) d \sigma \\
& +
\end{aligned}
$$

\section{Passo 4:
sistema alvo}

Considere a transformação backstepping de dimensão infinita do estado atrasado

$$
w(x, t)=u_{\mathrm{av}}(x, t)-k H\left[\tilde{\vartheta}_{\mathrm{av}}(t)+\int_{0}^{x} u_{\mathrm{av}}(\sigma, t) d \sigma\right],
$$

que mapeia o sistema (38)-(40) no sistema alvo:

$$
\begin{aligned}
\dot{\tilde{\vartheta}}_{\mathrm{av}}(t) & =k H \tilde{\vartheta}_{\mathrm{av}}(t)+w(0, t), \\
w_{t}(x, t) & =w_{x}(x, t), \quad x \in[0, D], \\
w(D, t) & =-\frac{1}{c} \partial_{t} u_{\mathrm{av}}(D, t) .
\end{aligned}
$$

onde na última linha simplesmente foram zeradas todas as funçóes seno e cosseno de $\omega, 2 \omega, 3 \omega$ and $4 \omega$ com média zero. do mais, o filtro $c /(s+c)$ também foi representado na forma em espaço de estado. A solução da EDP de

$$
u_{\mathrm{av}}(x, t)=U_{\mathrm{av}}(t+x-D) .
$$

Utlizando-se (42) para $x=D$ e o fato de que $u_{\mathrm{av}}(D, t)=$ $U_{\text {av }}(t)$, a partir de (45) obtém-se (40), i.e.,

$$
U_{\mathrm{av}}(t)=\frac{c}{s+c}\left\{k H\left[\tilde{\vartheta}_{\mathrm{av}}(t)+\int_{0}^{D} u_{\mathrm{av}}(\sigma, t) d \sigma\right]\right\} .
$$

Considere agora o sinal $w(D, t)$. É fácil mostrar que

$$
w_{t}(D, t)=\partial_{t} u_{\mathrm{av}}(D, t)-k H u_{\mathrm{av}}(D, t),
$$
onde $\partial_{t} u_{\mathrm{av}}(D, t)=\dot{U}_{\mathrm{av}}(t)$. A inversa de (42) é dada por

$$
\begin{aligned}
& u_{\mathrm{av}}(x, t)=w(x, t) \\
& +k H\left[e^{k H x} \tilde{\vartheta}_{\mathrm{av}}(t)+\int_{0}^{x} e^{k H(x-\sigma)} w(\sigma, t) d \sigma\right] .
\end{aligned}
$$

Substituindo-se (45) e (48) em (47), obtém-se

$$
\begin{aligned}
& w_{t}(D, t)=-c w(D, t)-k H w(D, t) \\
& -(k H)^{2}\left[e^{k H D} \tilde{\vartheta}_{\mathrm{av}}(t)+\int_{0}^{D} e^{k H(D-\sigma)} w(\sigma, t) d \sigma\right] .
\end{aligned}
$$

Passo 5: Funcional de Lyapunov-Krasovskii Considere o seguinte funcional de Lyapunov

$$
V(t)=\frac{\tilde{\vartheta}_{\mathrm{av}}^{2}(t)}{2}+\frac{a}{2} \int_{0}^{D}(1+x) w^{2}(x, t) d x+\frac{1}{2} w^{2}(D, t),
$$


onde o parâmetro $a>0$ será escolhido mais adiante. Temse que

$$
\begin{aligned}
& \dot{V}(t)=k H \tilde{\vartheta}_{\mathrm{av}}^{2}(t)+\tilde{\vartheta}_{\mathrm{av}}(t) w(0, t) \\
& +a \int_{0}^{D}(1+x) w(x, t) w_{x}(x, t) d x+w(D, t) w_{t}(D, t) \\
& =k H \tilde{\vartheta}_{\mathrm{av}}^{2}(t)+\tilde{\vartheta}_{\mathrm{av}}(t) w(0, t)+\frac{a(1+D)}{2} w^{2}(D, t) \\
& -\frac{a}{2} w^{2}(0, t)-\frac{a}{2} \int_{0}^{D} w^{2}(x, t) d x \\
& +w(D, t) w_{t}(D, t) \\
& \leq k H \tilde{\vartheta}_{\mathrm{av}}^{2}(t)+\frac{\tilde{\vartheta}_{\mathrm{av}}^{2}(t)}{2 a}-\frac{a}{2} \int_{0}^{D} w^{2}(x, t) d x \\
& +w(D, t)\left[w_{t}(D, t)+\frac{a(1+D)}{2} w(D, t)\right] .
\end{aligned}
$$

Lembrando-se que $k>0$ e $H<0$, escolhe-se

Então,

$$
a=-\frac{1}{k H} \text {. }
$$

$$
\begin{aligned}
& \dot{V}(t) \leq \frac{k H}{2} \tilde{\vartheta}_{\mathrm{av}}^{2}(t)+\frac{1}{2 k H} \int_{0}^{D} w^{2}(x, t) d x \\
& +w(D, t)\left[w_{t}(D, t)-\frac{(1+D)}{2 k H} w(D, t)\right] \\
& =-\frac{1}{2 a} \tilde{\vartheta}_{\mathrm{av}}^{2}(t)-\frac{a}{2} \int_{0}^{D} w^{2}(x, t) d x \\
& +w(D, t)\left[w_{t}(D, t)+\frac{a(1+D)}{2} w(D, t)\right] .
\end{aligned}
$$

Agora, considera-se (54) juntamente com (49). Completando-se os quadrados, obtém-se

$$
\begin{aligned}
& \dot{V}(t) \leq-\frac{1}{4 a} \tilde{\vartheta}_{\mathrm{av}}^{2}(t)-\frac{a}{4} \int_{0}^{D} w^{2}(x, t) d x \\
& +a\left|(k H)^{2} e^{k H D}\right|^{2} w^{2}(D, t) \\
& +\frac{1}{a}\left\|(k H)^{2} e^{k H(D-\sigma)}\right\|^{2} w^{2}(D, t) \\
& +\left[\frac{a(1+D)}{2}-k H\right] w^{2}(D, t)-c w^{2}(D, t) .
\end{aligned}
$$

Para escrevermos (55), utilizou-se das seguintes relações

$$
\begin{aligned}
& -w(D, t)\left\langle(k H)^{2} e^{k H(D-\sigma)}, w(\sigma, t)\right\rangle \\
& \leq|w(D, t)|\left\|(k H)^{2} e^{k H(D-\sigma)}\right\|\|w(t)\| \\
& \leq \frac{a}{4}\|w(t)\|^{2}+\frac{1}{a}\left\|(k H)^{2} e^{k H(D-\sigma)}\right\|^{2} w^{2}(D, t),
\end{aligned}
$$

onde a primeira desigualdade é a de Cauchy-Schwartz e a segunda é a de Young. A notação $\langle\cdot, \cdot\rangle$ denota o produto interno na variável espacial $\sigma \in[0, D]$, em que ambas $e^{k H(D-\sigma)}$ e $w(\sigma, t)$ dependem deste produto e $\|\cdot\|$ denota a norma $L_{2}$ em $\sigma$. Então, a partir de (55), chega-se a

$$
\begin{aligned}
\dot{V}(t) \leq & -\frac{1}{4 a} \tilde{\vartheta}_{\mathrm{av}}^{2}(t)-\frac{a}{4(1+D)} \int_{0}^{D}(1+x) w^{2}(x, t) d x \\
& -\left(c-c^{*}\right) w^{2}(D, t),
\end{aligned}
$$

onde

$c^{*}=\frac{a(1+D)}{2}-k H+a\left|(k H)^{2} e^{k H D}\right|^{2}+\frac{1}{a}\left\|(k H)^{2} e^{k H(D-\sigma)}\right\|^{2}$.

A partir de (58), é claro que um limitante superior $c^{*}$ pode ser obtido de limitantes inferiores e superiores da Hessiana desconhecida $H$ e do atraso $D$. Portanto, de (57), se $c$ é escolhido tal que $c>c^{*}$, obtém-se

$$
\dot{V}(t) \leq-\mu V(t)
$$

para algum $\mu>0$. Com isso, o sistema em malha fechada é exponencialmente estável no sentido da norma completa do estado

$$
\left(\left|\tilde{\vartheta}_{\mathrm{av}}(t)\right|^{2}+\int_{0}^{D} w^{2}(x, t) d x+w^{2}(D, t)\right)^{1 / 2},
$$

i.e., na variável transformada $\left(\tilde{\vartheta}_{\mathrm{av}}, w\right)$.

Passo 6: Estabilidade Exponencial (na norma $L_{2}$ ) para o Sistema em Malha Fechada Médio (38)-(40)

Para obter a estabilidade exponencial com relação à norma

$$
\left(\left|\tilde{\vartheta}_{\mathrm{av}}(t)\right|^{2}+\int_{0}^{D} u_{\mathrm{av}}^{2}(x, t) d x+u_{\mathrm{av}}^{2}(D, t)\right)^{1 / 2},
$$

é preciso mostrar que existem números positivos $\alpha_{1}$ e $\alpha_{2}$ tal que

$$
\alpha_{1} \Psi(t) \leq V(t) \leq \alpha_{2} \Psi(t),
$$

onde $\Psi(t):=\left|\tilde{\vartheta}_{\mathrm{av}}(t)\right|^{2}+\int_{0}^{D} u_{\mathrm{av}}^{2}(x, t) d x+u_{\mathrm{av}}^{2}(D, t)$, ou equivalentemente,

$$
\Psi(t):=\left|\tilde{\theta}_{\mathrm{av}}(t-D)\right|^{2}+\int_{t-D}^{t} U_{\mathrm{av}}^{2}(\tau) d \tau+U_{\mathrm{av}}^{2}(t),
$$

usando (37) e (41).

Isto é estabelecido diretamente a partir de (42), (48), (50) e empregando a desigualdade de Cauchy-Schwartz e outros cálculos, como na prova do Teorema 2.1 em (Krstić, 2009). Portanto, assim como (59), tem-se

$$
\Psi(t) \leq \frac{\alpha_{2}}{\alpha_{1}} e^{-\mu t} \Psi(0),
$$

que completa a prova da estabilidade exponencial.

\section{Passo 7: Otimilidade Inversa}

Baseando-se na prova do Teorema 6 em (Smyshlyaev and Krstic, 2004) e no Teorema 2.8 em (Krstic and Deng, 1999), no qual escolhe-se $c^{* *}=4 c^{*}$ e $c=2 c^{*}$ e defini-se $\mathcal{L}(t)$ como:

$$
\begin{aligned}
& \mathcal{L}(t)=-2 c \dot{V}(t)+c\left(c-4 c^{*}\right) w^{2}(D, t) \\
& \geq c\left(\frac{1}{2} k \tilde{\vartheta}_{\mathrm{av}}^{2}(t)+\frac{a}{2} \int_{0}^{D} w^{2}(x, t) d x+\left(c-2 c^{*}\right) w^{2}(D, t)\right),
\end{aligned}
$$

onde $\vartheta_{\mathrm{av}}(t):=\tilde{\theta}_{\mathrm{av}}(t-D)$.

Substituindo-se a transformação backstepping (49) na derivada do funcional de Lyapunov em (51), tem-se

$$
\begin{aligned}
& \dot{V}(t)=k H \tilde{\vartheta}_{\mathrm{av}}^{2}(t)+\tilde{\vartheta}_{\mathrm{av}}(t) w(0, t)+\frac{a(1+D)}{2} w^{2}(D, t) \\
& -\frac{a}{2} w^{2}(0, t)-\frac{a}{2} \int_{0}^{D} w^{2}(x, t) d x-2 c^{*} w^{2}(D, t) \\
& -k H w^{2}(D, t)-(k H)^{2} w(D, t) e^{k H D} \tilde{\vartheta}_{\mathrm{av}}(t) \\
& -(k H)^{2} w(D, t) \int_{0}^{D} e^{k H(D-\sigma)} w(\sigma, t) d \sigma,
\end{aligned}
$$


então $\mathcal{L}(t)$ será:

$$
\begin{aligned}
& \mathcal{L}(t)=-2 c k H \tilde{\vartheta}_{\mathrm{av}}^{2}(t)-2 c \tilde{\vartheta}_{\mathrm{av}}(t) w(0, t)-2 c \frac{a(1+D)}{2} w^{2}(D, t) \\
& +c a w^{2}(0, t)+c a \int_{0}^{D} w^{2}(x, t) d x+2 c k H w^{2}(D, t) \\
& +2 c(k H)^{2} w(D, t) e^{k H D} \tilde{\vartheta}_{\mathrm{av}}(t) \\
& +2 c(k H)^{2} w(D, t) \int_{0}^{D} e^{k H(D-\sigma)} w(\sigma, t) d \sigma+c^{2} w^{2}(D, t)
\end{aligned}
$$

No entanto substituindo-se a versão média do sistema (38) no sistema alvo em (43), tem-se

$$
u_{\mathrm{av}}(0, t)=k H \tilde{\vartheta}_{\mathrm{av}}(t)+w(0, t) .
$$

Isolando $w(0, t)$ em $(68)$ tem-se:

$$
w(0, t)=u_{\mathrm{av}}(0, t)-k H \tilde{\vartheta}_{\mathrm{av}}(t) .
$$

então substituindo-se (69) em (67), somando e subtraindo $\gamma \tilde{\vartheta}_{\mathrm{av}}^{2}(t)$ nos termos da equação, tem-se:

$$
\begin{aligned}
& \mathcal{L}(t)=c\left(a(k H)^{2} \tilde{\vartheta}_{\mathrm{av}}^{2}(t)-2(a k H+1) u_{\mathrm{av}}(0, t) \tilde{\vartheta}_{\mathrm{av}}(t)\right. \\
& -a(1+D) w^{2}(D, t)-\gamma \tilde{\vartheta}_{\mathrm{av}}^{2}(t)+2(k H)^{2} w(D, t) \\
& \left.\times\left[e^{k H D} \tilde{\vartheta}_{\mathrm{av}}(t)+\int_{0}^{D} e^{k H(D-\sigma)} w(\sigma, t) d \sigma\right]\right) \\
& +a u_{\mathrm{av}}^{2}(0, t)+\frac{a}{2} \int_{0}^{D} w^{2}(x, t) d x+w^{2}(D, t)\left(2 c^{*}+2 k H\right) \\
& +c\left(\gamma \tilde{\vartheta}_{\mathrm{av}}^{2}(t)+\frac{a}{2} \int_{0}^{D} w^{2}(x, t) d x+\left(c-2 c^{*}\right) w^{2}(D, t)\right)(70)
\end{aligned}
$$

sabe-se que $a=\frac{-1}{k H}$, então substituindo $k H$ por $\frac{-1}{a}$ em (70), tem-se

$$
\begin{aligned}
& \mathcal{L}(t)=c\left(\left[\frac{1}{a}-\gamma\right] \tilde{\vartheta}_{\mathrm{av}}^{2}(t)\left(2 c^{*}-a(1+D)-\frac{2}{a}\right) w^{2}(D, t)\right. \\
& +a u_{\mathrm{av}}^{2}(0, t)+\frac{a}{2} \int_{0}^{D} w^{2}(x, t) d x+\frac{2}{a^{2}} w(D, t) \\
& \left.\times\left[e^{k H D} \tilde{\vartheta}_{\mathrm{av}}(t)+\int_{0}^{D} e^{k H(D-\sigma)} w(\sigma, t) d \sigma\right]\right) \\
& +c\left(\gamma \tilde{\vartheta}_{\mathrm{av}}^{2}(t)+\frac{a}{2} \int_{0}^{D} w^{2}(x, t) d x+\left(c-2 c^{*}\right) w^{2}(D, t)\right)(71)
\end{aligned}
$$

Sendo que $\mathcal{L}(t)$ pode ser reescrito como:

$$
\mathcal{L}(t)=\Upsilon(D, t)+c\left(\gamma \tilde{\vartheta}_{\mathrm{av}}^{2}(t)+\frac{a}{2} \int_{0}^{D} w^{2}(x, t) d x+\left(c-2 c^{*}\right) w^{2}(D, t)\right) .
$$

No qual $\Upsilon(D, t)$ é dado por:

$$
\begin{aligned}
& \Upsilon(D, t)=c\left(\left[\frac{1}{a}-\gamma\right] \tilde{\vartheta}_{\mathrm{av}}^{2}(t)+\left(2 c^{*}-a(1+D)-\frac{2}{a}\right) w^{2}(D, t)\right. \\
& +a u_{\mathrm{av}}^{2}(0, t)+\frac{a}{2} \int_{0}^{D} w^{2}(\sigma, t) d \sigma+\frac{2}{a^{2}} w(D, t) e^{k H D} \tilde{\vartheta}_{\mathrm{av}}(t) \\
& \left.+\frac{2}{a^{2}} w(D, t) \int_{0}^{D} e^{k H(D-\sigma)} w(\sigma, t) d \sigma\right)
\end{aligned}
$$

Para satisfazer a inequação em (65), é necessário garantir $\Upsilon(D, t) \geq 0$. Para satisfazer essa condição, serão analisados os termos não quadráticos presentes em (73) de modo que se assegure que estes sejam iguais ou maiores que zero. Após a adição e subtração de termos em $(73), \Upsilon(D, t)$ pode ser reescrito como:

$$
\begin{aligned}
& \Upsilon(D, t)=c\left(\left[\frac{1}{a}-\frac{1}{a^{2}}-\gamma\right] \tilde{\vartheta}_{\mathrm{av}}^{2}(t)\right. \\
& +\left(2 c^{*}-a(1+D)-\frac{2}{a}-\frac{1}{a^{2}}-\frac{2 \sqrt{D}}{a^{2}}\right) w^{2}(D, t) \\
& +a u_{\mathrm{av}}^{2}(0, t)+\left[\frac{a}{2}-\frac{2 \sqrt{D}}{a^{2}}\right] \int_{0}^{D} w^{2}(\sigma, t) d \sigma \\
& +\frac{2}{a^{2}} w(D, t) e^{k H D} \tilde{\vartheta}_{\mathrm{av}}(t)+\frac{1}{a^{2}} w^{2}(D, t)+\frac{1}{a^{2}} \tilde{\vartheta}_{\mathrm{av}}^{2}(t) \\
& +\frac{2}{a^{2}} w(D, t) \int_{0}^{D} e^{k H(D-\sigma)} w(\sigma, t) d \sigma \\
& \left.+\frac{2 \sqrt{D}}{a^{2}} w^{2}(D, t)+\frac{2 \sqrt{D}}{a^{2}} \int_{0}^{D} w^{2}(\sigma, t) d \sigma\right) .
\end{aligned}
$$

Através das desigualdades de Young e Cauchy-Schwartz é possível verificar o majorante dos termos adicionados e subtraidos em (74), de modo que:

$$
\begin{gathered}
\frac{1}{a^{2}} w^{2}(D, t)+\frac{1}{a^{2}} \tilde{\vartheta}_{\mathrm{av}}^{2}(t) \geq \frac{2}{a^{2}}\left|w(D, t) e^{k H D} \tilde{\vartheta}_{\mathrm{av}}(t)\right| \\
\frac{2 \sqrt{D}}{a^{2}}\left(w^{2}(D, t)+\int_{0}^{D} w^{2}(\sigma, t) d \sigma\right) \\
\geq \frac{2}{a^{2}}\left|w(D, t) \int_{0}^{D} e^{k H(D-\sigma)} w(\sigma, t) d \sigma\right|
\end{gathered}
$$

Analisando $\Upsilon(D, t)$, pelos limitantes inferiores, tem-se

$$
\begin{aligned}
& \Upsilon(D, t) \geq c\left(\left[\frac{1}{a}-\frac{1}{a^{2}}-\gamma\right] \tilde{\vartheta}_{\mathrm{av}}^{2}(t)\right. \\
& +\left(2 c^{*}-a(1+D)-\frac{2}{a}-\frac{1}{a^{2}}-\frac{2 \sqrt{D}}{a^{2}}\right) w^{2}(D, t) \\
& +a u_{\mathrm{av}}^{2}(0, t)+\left[\frac{a}{2}-\frac{2 \sqrt{D}}{a^{2}}\right] \int_{0}^{D} w^{2}(\sigma, t) d \sigma \\
& +\frac{2}{a^{2}} w(D, t) e^{k H D} \tilde{\vartheta}_{\mathrm{av}}(t)+\frac{2}{a^{2}}\left|w(D, t) e^{k H D} \tilde{\vartheta}_{\mathrm{av}}(t)\right| \\
& +\frac{2}{a^{2}} w(D, t) \int_{0}^{D} e^{k H(D-\sigma)} w(\sigma, t) d \sigma \\
& \left.+\frac{2}{a^{2}}\left|w(D, t) \int_{0}^{D} e^{k H(D-\sigma)} w(\sigma, t) d \sigma\right|\right) .
\end{aligned}
$$

Logo, para garantir $\Upsilon(D, t) \geq 0$ é necessário seguir as seguintes condições:

\section{Condição:}

$$
\begin{array}{r}
\frac{1}{a}-\frac{1}{a^{2}}-\gamma>0 \\
\gamma<\frac{a-1}{a^{2}}
\end{array}
$$

2 Condição: Sabendo que $c=2 c^{*}$

$$
\begin{array}{r}
2 c^{*}-a(1+D)-\frac{2}{a}-\frac{1}{a^{2}}-\frac{2 \sqrt{D}}{a^{2}}>0 \\
c>a(1+D)+\frac{2}{a}+\frac{1}{a^{2}}+\frac{2 \sqrt{D}}{a^{2}}
\end{array}
$$




\section{Condição:}

$$
\begin{array}{r}
\frac{a}{2}-\frac{2 \sqrt{D}}{a^{2}}>0 \\
a>\sqrt[3]{4 \sqrt{D}}
\end{array}
$$

Assim sendo, então $\mathcal{L}(t)$ dado em $(72)$ e $\Upsilon(D, t)$ dado em (73), perante as condições impostas para $\gamma, a$ e $c$, pode-se afirmar que $\Upsilon(D, t) \geq 0$, logo:

$$
\mathcal{L}(t) \geq c\left(\frac{1}{2} k \tilde{\vartheta}_{\mathrm{av}}^{2}(t)+\frac{a}{2} \int_{0}^{D} w^{2}(x, t) d x+\left(c-2 c^{*}\right) w^{2}(D, t)\right)
$$

$\operatorname{com} \gamma=k / 2$.

Ou seja $\mathcal{L}(t) \geq \mu \Psi(t)^{2}$, pela mesma razão que mantém a condição vista em (62), completando a prova da otimalidade inversa.

Teorema 2. Considere o sistema em malha fechada da Figura (1), com saída atrasada (3). Existe $c^{*}>0$ tal que, $\forall c \geq c^{*}, \exists \omega^{*}(c)>0$ tal que, $\forall \omega>\omega^{*}$, o sistema atrasado em malha fechada (5) e (19), com $G(t)$ em (6), $\hat{H}(t)$ em (8) e estado $\tilde{\theta}(t-D), U(\sigma), \forall \sigma \in[t-D, t]$, tem uma única solução periódica exponencialmente estável em $t$ de período $\Pi=2 \pi / \omega$, denotada por $\tilde{\theta}^{\Pi}(t-D), U^{\Pi}(\sigma), \forall \sigma \in[t-D, t]$, satisfazendo, $\forall t \geq 0$ :

$$
\left(\left|\tilde{\theta}^{\Pi}(t-D)\right|^{2}+\left[U^{\Pi}(t)\right]^{2}+\int_{t-D}^{t}\left[U^{\Pi}(\tau)\right]^{2} d \tau\right)^{1 / 2}
$$

Além disso,

$$
\begin{aligned}
& \limsup _{t \rightarrow+\infty}\left|\theta(t)-\theta^{*}\right|=\mathcal{O}(a+1 / \omega), \\
& \limsup _{t \rightarrow+\infty}\left|y(t)-y^{*}\right|=\mathcal{O}\left(a^{2}+1 / \omega^{2}\right) .
\end{aligned}
$$

A prova do Teorema 2, pode ser obtida através dos passos 6 e 7 do Teorema 1 em (Oliveira et al., 2017)

\section{SIMULAÇÕES}

Para avaliar o efeito da otimalidade inversa no controle por busca extremal com atraso, o seguinte mapeamento quadrático é considerado

$$
Q(\theta)=5-(0.1)(\theta-3)^{2},
$$

com um atraso de $D=5 \mathrm{~s}$. De acordo com (82), o ponto de extremo é $\left(\theta^{*}, y^{*}\right)=(3 ; 5)$ e a Hessiana do mapeamento estático é $H=-0,2$. Para a simulação foram utilizados os seguintes parâmetros: $\omega=10 \mathrm{rad} / \mathrm{s}, k=0,8, \theta(0)=-5 \mathrm{e}$ $a=0,2$, o valor de $c$ foi escolhido conforme imposto pela condição 2 , sendo $c=40$.

A Figura 2 apresenta uma comparação entre as variáveis fundamentais do ESC, com e sem a utilização do filtro $\frac{c}{s+c}$ na lei de controle (19). Como pode-se observar, no primeiro caso onde a otimalidade inversa é garantida, a convergência acontece de modo uniforme.

\section{CONCLUSÃO}

A estratégia de busca extremal via preditor com estimativa da Hessiana baseada em perturbação senoidal apresentada neste trabalho foi capaz de garantir ao sistema em malha fechada propriedades de estabilidade mesmo na presença de atrasos nos atuadores e sensores. A estabilidade exponencial e a convergência ao ponto de extremo foram rigorosamente demonstradas. Uma melhora significativa pode ser observada com a utilização da otimalidade inversa, pois além dos resultados teóricos, simulações ilustraram uma considerável suavização da busca do ponto de extremo, a qual resultou na eliminação de perturbação no transitório do sinal de saída e em uma diminuição no esforço de controle. Embora o atraso tenha sido considerado ao longo deste artigo, a otimalidade inversa também pode ser empregada em sistemas cujas plantas não possuem atrasos. Para trabalho futuro, indica-se o estudo da otimalidade inversa para atrasos desconhecidos e para sistemas com múltiplas entradas.

\section{REFERÊNCIAS}

Adetola, V. and Guay, M. (2007). Guaranteed parameter convergence for extremum-seeking control of nonlinear systems. Automatica, 43, 105-110.

Ghaffari, A., Krstić, M., and Nesić, D. (2012). Multivariable Newtonbased extremum seeking. Automatica, 1759-1767.

Hale, J.K. and Lunel, S.M.V. (1990). Averaging in infinite dimensions. Journal of Integral Equations and Applications, 2, 463-494.

Kalman, R.E. (1964). When is a linear control system optimal? ASME, J. Basic Eng., 86, 51-61.

Krstić, M. (2008). Lyapunov tools for predictor feedbacks for delay systems: inverse optimality and robustness to delay mismatch. Automatica, 44, 2930-2935.

Krstić, M. (2009). Delay Compensation for Nonlinear, Adaptive, and PDE Systems. Birkhaüser.

Krstić, M. (2014). Extremum seeking control. in T. Samad and J. Baillieul, Encyclopedia of Systems and Control, Springer.

Krstic, M. and Deng, H. (1999). Stabilization of Nonlinear Uncertain Systems. Springer.

Krstić, M. and Wang, H.H. (2000). Stability of extremum seeking feedback for general nonlinear dynamic systems. Automatica, 595601.

Lehman, B. (2002). The influence of delays when averaging slow and fast oscillating systems: overview. IMA Journal of Mathematical Control and Information, 19, 201-215.

Liu, S.J. and Krstić, M. (2012). Stochastic Averaging and Stochastic Extremum Seeking. Springer.

Nesić, D., Tan, Y., Moase, W., and Manzie, C. (2010). A unifying approach to extremum seeking: adaptive schemes based on the estimation of derivatives. IEEE Conference on Decision and Control, Atlanta, 4625-4630.

Oliveira, T.R., Hsu, L., and Peixoto, A.J. (2011). Output-feedback global tracking for unknown control direction plants with application to extremum-seeking control. Automatica, 2029-2038.

Oliveira, T.R., Krstic, M., and Tsubakino, D. (2017). Extremum seeking for static maps with delays. IEEE Trans. Automat. Contr., 62(4), 1911--1926.

Oliveira, T.R., Peixoto, A.J., and Hsu, L. (2012). Global real-time optimization by output-feedback extremum-seeking control with sliding modes. Journal of The Franklin Institute, 1397-1415.

Smyshlyaev, A. and Krstic, M. (2004). Closed form boundary state feedbacks for a class of 1-D partial integro-differential equations. IEEE Transactions on Automatic Control, 49(12), 2185-2202.

Tan, Y., Nesić, D., Mareels, I.M.Y., and Astolfi, A. (2009). On global extremum seeking in the presence of local extrema. Automatica, 45, 245-251. 

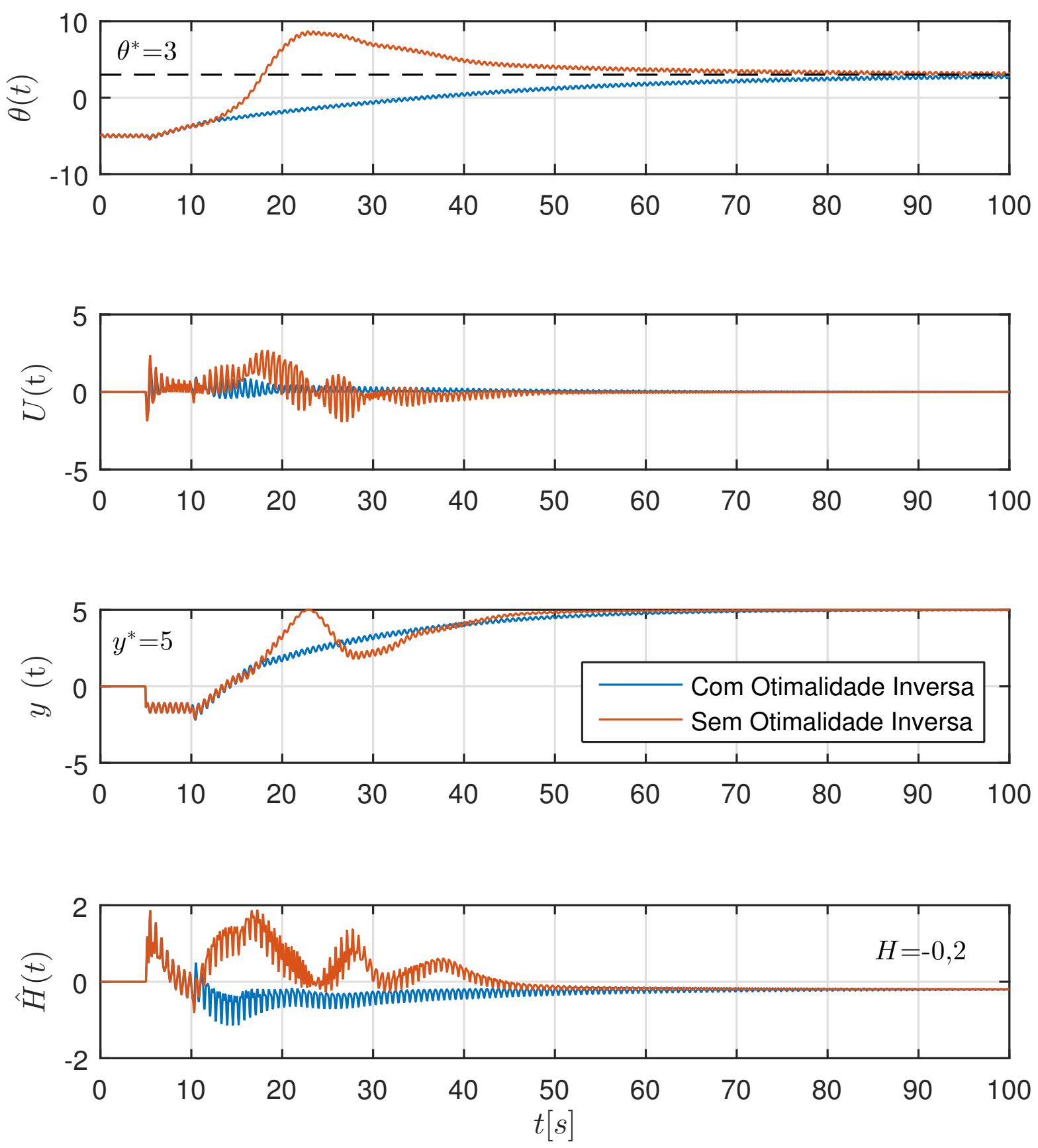

Figura 2. ESC com e sem otimalidade inversa: (a) parâmetro $\theta(t)$ (sinal de entrada); (b) sinal de controle $U(t)$;

(c) sinal de saída $y(t)$ e (d) estimativa da Hessiana $\hat{H}(t)$. 